\title{
EFFECT OF DIETHYLENETRIAMINE AND TRIETHYLAMINE SENSITIZATION ON THE CRITICAL DIAMETER OF NITROMETHANE
}

\author{
J.J. Lee ${ }^{*}$ J. Jiang ${ }^{\dagger}$, K.H. Choong ${ }^{\dagger}$, J.H.S. Lee ${ }^{\dagger}$ \\ *Graduate Aeronautics Laboratory, California Institute of Technology, Pasadena, CA 91125, LISA \\ ${ }^{\dagger}$ Dept. of Mechanical Engineering, McGill University, Montréal, Québec, Canada, H3A 2K6
}

\begin{abstract}
In this work, the critical diameter for detonation was measured for Nitromethane (NM) sensitized with two different amines: Diethylenetriamine (DETA) and Triethylamine (TEA). The critical diameter in glass and polyvinylchloride tubes is found to decrease rapidly as the amount of sensitizer is increased, then increase past a critical amount of sensitizer. Thus the critical diameter reaches a minimum at a critical concentration of sensitizer. It was also found that the critical diameter is lower with DETA than with TEA.
\end{abstract}

\section{INTRODUCTION}

Previous studies have shown that small concentrations of certain substances can strongly increase the explosive sensitivity nitromethane (NM). Amines are found to be the most effective chemical sensitizing agent for NM with ethylenediamine and diethylenetriamine (DETA) producing the largest increase in the card gap value (1). Triethylamine (TEA) is favored in certain lowtemperature applications on account of it's freezing point of $-115^{\circ} \mathrm{C}$.

Although the card gap method has been used to compare various sensitizers, the critical diameter is a more fundamental dynamic parameter for detonation since it is directly related to the chemical length scale of the detonation wave (2). In gaseous detonations, the critical diameter is directly proportional to the cell size parameter. The cell size has been used to formulate many semi-empirical correlations such as the critical energy for direct initiation, the critical tube diameter for detonation propagation in various tubes and channels, and the critical conditions for propagation in porous media (3).

The effect of DETA on the critical diameter of NM has been reported by Engelke (4), who performed measurements with up to $2.5 \%$ DETA by mass in NM. Engelke observed a reduction in the critical diameter of over $50 \%$ in the range of DETA concentrations used. In this work, the critical diameter measurements in NM are extended for DETA concentrations up to $20 \%$ by mass. The critical diameter of NM sensitized with up to $15 \%$ TEA by mass is also measured.

\section{EXPERIMENTAL DETAILS}

The critical diameter was measured in tubes of glass or polyvinylchloride (Tygon $^{\mathrm{TM}}$ ). Tubes of different diameter with a length of $150 \mathrm{~mm}$ were attached at one end to a $3 \mathrm{cc}$ plastic syringe with a length of $70 \mathrm{~mm}$ and a diameter of $8 \mathrm{~mm}$, as shown in 


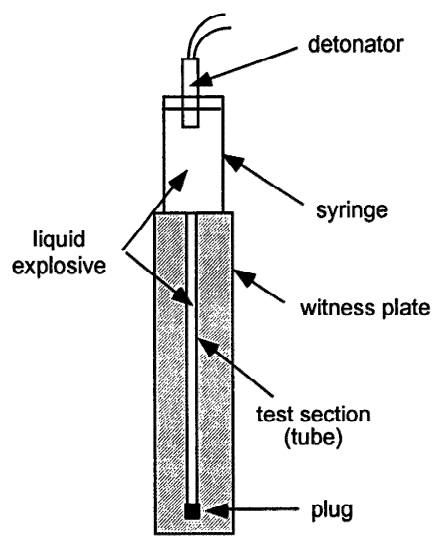

FIGURE 1. Apparatus used for critical diameter measurements of liquid explosives.

Fig. 1. The liquid explosive in the syringe-tube assembly is initiated by an electric blasting cap inserted into the syringe end. A steel or aluminium witness plate placed against the tube was used to determine whether the detonation had propagated the full length of the tube or not.

NM with different concentrations of sensitizer was tested in tubes of different diameter in order to determine the critical diameter. In certain cases, the critical diameter was measured by using a fixed tube diameter and varying the sensitizer concentration until super-critical and sub-critical concentrations were found. This latter procedure was chosen when high accuracy was desired within a certain range of sensitizer concentrations where tubes were not available in fine enough increments of diameter.

The witness plate method provided a clear indication of the distance of propagation of the detonation along the tube, and non-ambiguously showed whether the tube diameter was above or below the critical value. Since the tube length was over 20 times the diameter, there was sufficient distance for the detonation to reach a steady state. Velocity measurements performed in a previous work (6) showed that it was uniform along the tube for super-critical diameters, hence confirming the stability of the wave.

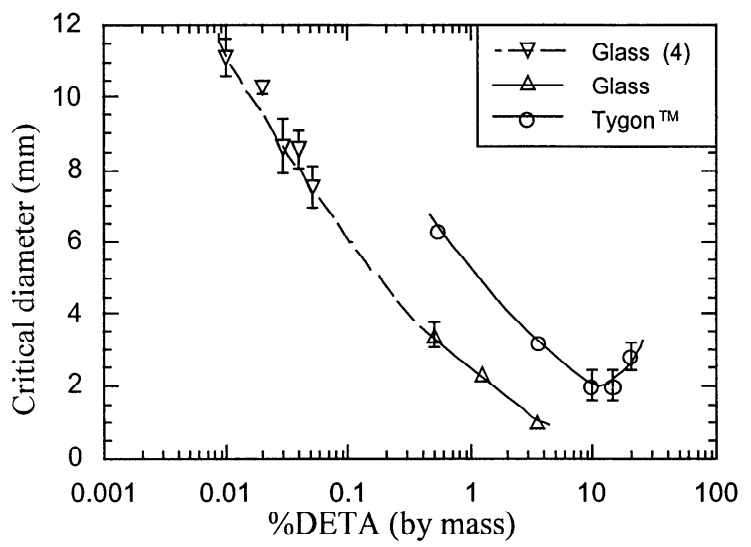

FIGURE 2. Critical diameter on NM sensitized with DETA in glass and Tygon ${ }^{\mathrm{TM}}$ tubes. Error bars are not shown when they are smaller than the data point symbol.

\section{RESULTS AND DISCUSSION}

The critical diameter for NM was found to decrease sharply with only a small concentration of DETA added. At a concentration of $0.5 \%$ DETA, the critical diameter in glass tubes decreased $0.3 \mathrm{~mm}$ from its value of $16.2 \mathrm{~mm}$ for pure NM (4), as seen in Fig. 2. The present results extrapolate well to Engelke's results with DETA concentrations less than $0.053 \%$.

This strong reduction of the critical diameter is an indication of a decreased reaction zone length in the detonation front, or equivalently, an increase in the rcaction rate. This can occur when the amine chemically weakens certain bonds in the NM molecule, effectively reducing the activation energy (7). Independent observations of another ratedependent parameter, the time-to-detonation in a shocked explosive, also support a strong increase in the reaction rate of NM with the addition of minute amounts of DETA (5). Hence the addition of DETA effectively controls the characteristic chemical time scale of the detonation front.

Also apparent from the results (Fig. 2) is that the critical diameter of liquid explosive confined in Tygon $^{\mathrm{TM}}$ is higher than with glass. This can also be seen in the critical diameter results for NM sensitized with TEA (Fig. 3). This can be explained by the stronger confinement of glass on the explosive due to its higher shock impedance. The 
effect of confinement on the detonation properties has been recently discussed in Brouillette et al. (6).

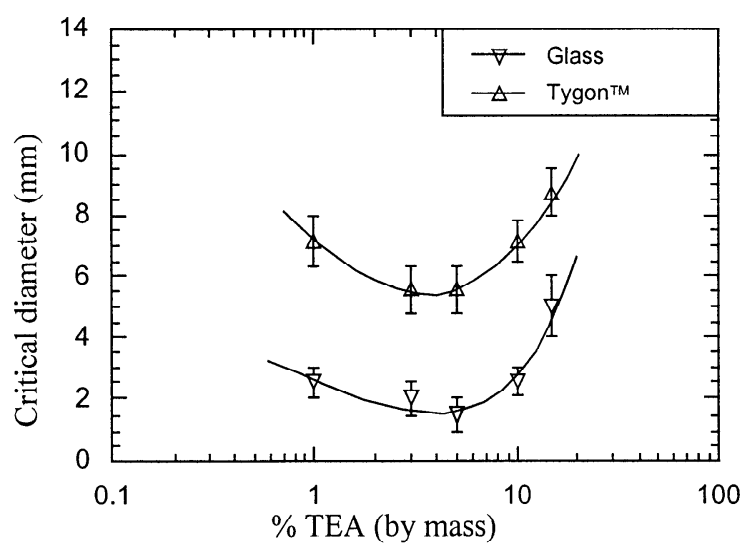

FIGURE 3. The critical diameter of NM sensitized with TEA in glass and Tygon ${ }^{\mathrm{TM}}$ tubes.

One of the main features of the critical diameter dependence on the concentration of sensitizer is a minimum critical diameter which occurs at a critical sensitizer concentration. As the concentration of sensitizer increases past the critical value, the critical diameter begins to increase sharply. This phenomenon is once again present with both DETA and TEA mixtures (Figs. 2 and 3). The minimum diameter occurs in DETA-sensitized mixtures at around $15 \%$ for Tygon tubes. Although the minimum was not reached for glass tubes (Fig. 2), it would likely occur at the same DETA concentration as for Tygon tubes (\%15) since the sensitizer concentration at the minimum does not seem to be influenced by the confinement (Fig. 3). For TEA mixtures, the minimum diameter occurs around $5 \%$.

A possible explanation for the minimum in the critical diameter curve may be deduced from the dilution effect of the sensitizer. As the concentration of sensitizer increases, the energy released in the detonation wave decreases as does the temperature in the wave. This effectively reduces the reaction rate and increases the critical diameter. At small sensitizer concentrations, the critical diameter decreases due to a reduction in the activation energy, however at larger concentrations, the dilution effect takes over and increases the critical diameter.

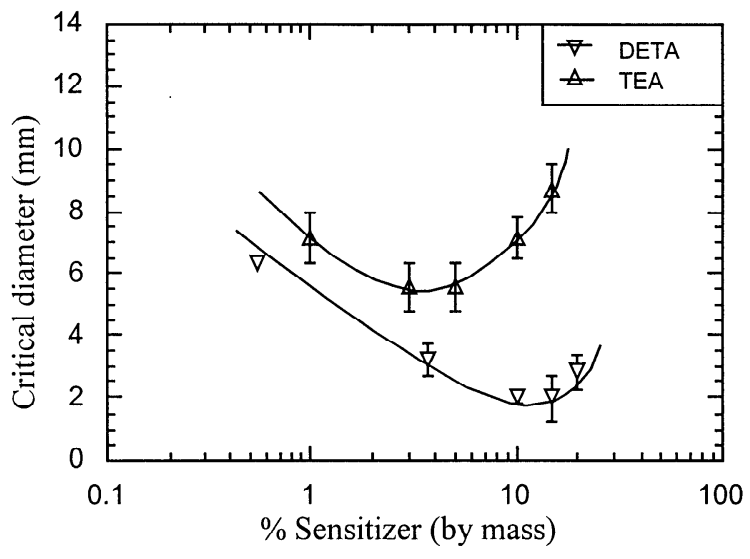

FIGURE 4. The critical diameter of NM sensitized with DETA and TEA in Tygon ${ }^{\mathrm{TM}}$ tubes.

This is further supported by comparing the sensitizing effect of both amines. As seen in Fig. 4, the critical diameter of NM is smaller for DETA sensitization than with TEA. Hence DETA is a more effective sensitizer. Furthermore, the critical diameter minimum for DETA mixtures is shifted towards higher concentrations as compared to TEA mixtures. Since the sensitizing effect is stronger with DETA, a larger decrease in temperature is necessary to counteract the effect of sensitization. Consequently, the minimum occurs at a higher concentration where the dilution effect is stronger.

\section{CONCLUSION}

The addition of DETA and TE $\Lambda$ to NM has been found to strongly reduce the critical diameter of the liquid explosive, with DETA producing a stronger reduction. At higher concentrations of the sensitizer, the critical diameter increases sharply, producing a minimum in the critical diameter dependence on sensitizer concentration. This minimum results from a competition between the sensitizing effect and the dilution effect of the sensitizers. 


\section{REFERENCES}

1. Murray, S.B., Briosi, G.K., Pinco M.E., Anderson C.J., DRES Suffield Report No. 601, (1994).

2. Lee, J.II.S, "Dynamic paramctcrs of gascous detonation", Ann. Rev. Fluid Mech. 16: 311-336 (1984).

3. Makris, A., "The propagation of gaseous detonation in porous media", Ph.D. Thesis, McGill University, Canada, (1993) .

4. Engelke, R., "Effect of a chemical inhomogeneity on steady-state detonation velocity", Phys. Fluids 23(5):875880 (1980).

5. Walker, F.E., "Initiation and detonation studies in sensitized nitromethane", Acta Astronautica 6: 807-813 (1979).

6. Brouillette, M., Dionne, J.-P., Radulescu, M., Jiang, J., Lee, J.J., Lee J.H.S., "Detonation properties in confined liquid explosives", Proceedings of the 2Ist Int'l Symp. on Shock Waves (on CD), Ed. A.F.P. Howing, ISBN 0-90968316-6, Paper 3400, (1997).

7. Engelke, R., Earl, W.L., McMichael Rohling, C., "Microscopic evidence that the nitromethane aci ion is a rate controlling species in the detonation of liquid nitromethane", J. Chem. Phys.84(1): 142-146 (1986). 